\title{
Cardiac Dysfunction in Cirrhotic Patients
}

\author{
Khairy H. Morsy', Amr M. Zaghloul ${ }^{1}$, Mohamed E. Ahmed², Mostafa M. \\ Mostafa $^{1}$ \\ Departments of Tropical medicine and Gastroenterology ${ }^{1}$ and Internal \\ medicine $^{2}$,Sohag Faculty of Medicine, Sohag University
}

\begin{abstract}
Introduction:In the last 20 years a number of evidences suggested that cirrhosis regardless of its etiology, is associated with major cardiovascular anomalies. Overall these alterations are known as cirrhotic cardiomyopathy and a wide range of cardiovascular abnormalities including hyperdynamic circulation, enlargement or hypertrophy of different cardiac chambers and electrophysiological changes such as QT prolongation.

Aim of the work:to study the pattern and the extent of cardiac affection in cirrhotic patients and its relation to the Child-Pugh classification and its relation to MELD score.

Patients and Methods: Our study was carried out on 45 patients with liver cirrhosis, the diagnosis was depending on clinical evidence of stigmata of chronic liver disease (e.g.jaundice, ascites, etc), Ultrasonographic features of liver cirrhosis (e.g coarse echo texture, shrunken liver, etc) and laboratory investigations. Our study included 15 healthy controls. All patients were subjected to the complete history taking and physical examination, laboratory investigations: Aspartate aminotransferase (AST), Alanine aminotransferase (ALT), serum alkaline phosphatase, serum albumin, Prothrombin time and concentration, Total, direct bilirubin, hepatitis markers for $\mathrm{HBV}$ and $\mathrm{HCV}$, blood sugar, $\mathrm{Hb}$, serum Creatinine, serum Sodium and serum Potassium, echo Doppler study included chamber sizes, interventricular septum diameter, ejection fraction and diastolic function. All patients were classified according to the child-pugh classification and were be scored according to MELD score.
\end{abstract}

Results:In our study, only $22 \%$ of cases had no diastolic dysfunction, compared to $67 \%$ of controls. Also, $13.3 \%$ of cases had grade 2 diastolic dysfunction, compared to zero among controls. The difference was statistically significant. Diastolic dysfunction was significantly higher among Child class B and C compared to A $(\mathrm{p}=0.020)$. Although MELD increases steadily with second grade diastolic dysfunction, the difference was non significant. Diastolic dysfunction was more common among patients with larger amont of ascites, with significant difference. HE was significantly associated with diastolic dysfunction.

Conclusion:Our study concluded that diastolic dysfunction is an important pathological complication associated with liver cirrhosis and is directly related to the severity of liver cirrhosis.

Keywords: liver cirrhosis, cirrhotic cardiomyopathy, systolic and diastolic dysfunction.

\section{Introduction}

In the last 20 years a number of evidences suggested that cirrhosis whatever its etiology, is associated with major cardiovascular anomalies. Overall these alterations are known as cirrhotic cardiomyopathy ${ }^{(1,2)}$ and a wide range of cardiovascular abnormalities including hyperdynamic circulation, enlargement or hypertrophy of different cardiac 
chambers and electrophysiological changes such as QT prolongation $^{(3)}$.Cirrhosis is a state in which the liver slowly deteriorates and is unable to function normally due to chronic, or long lasting, injury ${ }^{(4)}$.The main causes in developed countries are infection with hepatitis $\mathrm{C}$ virus, alcohol misuse, and, increasingly, non-alcoholic liver disease; infection with hepatitis B virus is the most common cause in sub Saharan Africa and most parts of Asia ${ }^{(5)}$.

Clinicaly, several people with cirrhosis have no symptoms in the early stages of the disease. However, as the disease develop, a person experience several symptoms as fatigue, feeling tired, weakness, itching, loss of appetite, weight loss, nausea, bloating of the abdomen from ascites, lower limbs edema, spiderlike blood vessels, called spider angiomas, on the skin or jaundice. As the liver fails, complications may develop as portal hypertension ${ }^{(6)}$ that may leads to splenomegaly that may be complicated by hypersplenism, varices whatever esophageal, gastric or at any site that may be complicated by acute gastrointestinal bleeding $^{(7)}$,edema and ascites that may be complicated by spontaneous bacterial peritonitis or hepaticencephalopathy that manifested by confusion, personality changes and memory loss ${ }^{(7)}$. Also, liver cirrhosis may be complicated by liver cancerwith high mortality rate ${ }^{(8)}$. Cardiomyopathy is a state where the heart muscle is abnormal. Cardiomyopathy makes it harder for heart to pump and deliver blood to the rest of the body which may lead to heart failure. Cardiomyopathy can be treated according to the type of cardiomyopathy and its severity. Treatment may include medications, surgically implanted devices or, in severe cases, a heart transplant ${ }^{(9)}$.
Cirrhotic cardiomyopathy (CCM) is a form of chronic cardiac dysfunction in patients with cirrhosis, characterized by blunted contractile responsiveness to stress, and/or altered diastolic relaxation with electrophysiological abnormalities in the absence of other known cardiac disease. It is diagnosed if there is evidence of either systolic, or diastolic dysfunction, together with supporting criteria such as electrophysiological abnormalities or abnormal serum markers are present ${ }^{(10)}$. Different mechanisms are involved in the pathogenesis of cirrhotic cardiomyopathy, especially increased cardiac output related to hyperdynamic state of circulation, increased vasodilator production and activity, such as NO and decreased metabolization of other vasodilators due to liver failure and portosystemic shunt. These aspects contribute to decrease peripheral vascular resistance, hyperdynamic circulation and insufficient cardiac output for metabolic demand $^{(11)}$.

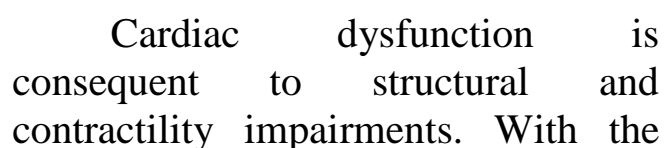
progression of liver disease, the patients often present symptoms only in situations of physiological and/or pharmacological stress ${ }^{(12)}$. It is also worth noting that the decrease in systolic and diastolic functions may cause heart failure with decrease left ventricular ejection fraction and is a determining factor for the development of hepatorenal syndrome. Diastolic dysfunction usually precedes systolic dysfunction, which is observed in situations of increased $\mathrm{CO}$ demand associated with decreased myocardial contractility ${ }^{(12)}$. Cardiac Doppler echocardiography in patients with CCM may show decrease and/or reversal of E/A ratio $(<1)$ and prolonged $\mathrm{E}$ wave 
deceleration time and isovolumetric relaxation time. These findings are suggestive of ventricular relaxation delay and, therefore, diastolic impairment. Initial studies on global longitudinal strain (GLS) have shown that this parameter is effective for detecting systolic and diastolic dysfunction, being far superior in detecting systolic disorders at rest ${ }^{(13)}$. It has been determined that the most specific marker for detecting diastolic dysfunction is early diastolic mitral annulus velocity $\left(e^{6}\right)^{(13)}$. Also, ejection fraction is a key measure to assess systolic function ${ }^{(14)}$. Most cases of cirrhotic cardiomyopathy not clinically obvious and generally the patients are asymptomatic. When cardiac failure occurs following stress, management should follow similar guidelines as in non-cirrhotic patients. However, cardiac after-load reduction will not be well tolerated in patients with advanced cirrhosis who are already significantly vasodilated. With a better understanding of the pathophysiology of cirrhotic cardiomyopathy, there are now a few scattered reports on the treatment of this condition. The removal of cardiotoxins using some form of liver dialysis is also a possibility ${ }^{(15)}$.

\section{Aim of the work:}

To study the pattern and the extent of cardiac affection in cirrhotic patients and its relation to the childpugh classification and its relation to MELD score.

\section{Patients and Methods:}

Our study was carried out on 45 patients with liver cirrhosis diagnosed depending on clinical evidence of stigmata of chronic liver disease (e.g.jaundice, ascites, etc) and ultrasonographic features of liver cirrhosis (e.g coarse echo texture, shrunken liver, etc) and laboratory investigation.our study included 15 healthy controls. Before starting our study, the protocol was approved by faculty ethics committee. All participants signed written consents.All patients that were included in our study are cirrhotic and were classified according to the childpugh classification by clinical and sonographic evaluation of ascites, clinical evaluation of encephalopathy and Laboratory evaluation ofserum bilirubin, serum albumin and INR, and were be scored according to MELD score by Laboratory evaluation of serum bilirubin, serum creatinine, and the international normalized ratio for prothrombin time (INR) and calculated the following formula: MELD $=0,378 \times \ln [$ serum bilirubin $(\mathrm{mg} / \mathrm{dL})]+11.2 \times \ln [\mathrm{INR}]+$ $0,957 \times \ln [$ serum creatinine $(\mathrm{mg} / \mathrm{dL})]+$ $0,643 \times 10$.

Patients with cardiovascular diseases (e.g.hypertension, ischemic heart disease, valvular heart disease,major arrhythmias), patients with severe anemia(haemoglobin below $7 \mathrm{gm} / \mathrm{dl}$ ), patients with major lung diseases, patients with renal failure(serum Creatinine more than $1.5 \mathrm{mg} / \mathrm{dl}$ ), patients with acute gastrointestinal bleeding,recent therapeutic paracentesis, patients with Hepatocellular carcinoma(beyond Milan criteria), and patients with diabetes mellitus were excluded from our study.

\section{Methods:}

Each patients included in the study were subjected to complete history taking, physical examination, laboratory investigations as Aspartate aminotransferase (AST), Alanine aminotransferase (ALT), serum alkaline phosphatase, serum albumin, Prothrombin time and concentration, Total, direct bilirubin, hepatitis markers for $\mathrm{HBV}$ and $\mathrm{HCV}$, blood sugar, $\mathrm{Hb}$, serum Creatinine, serum Sodium and serum Potassium, abdominal ultrasonography, echo 
Doppler study included chamber sizes, interventricular septum diameter, ejection fraction and diastolic function.

\section{Statistical analysis:}

- Statistical package for social sciences (IBM-SPSS), version 24 IBM- Chicago, USA (May 2016) was used for statistical data analysis.

- Data expressed as mean, standard deviation (SD), number and percentage. Mean and standard deviation were used as descriptive value for quantitative data, while number and percentage were used to describe qualitative data.

- Student $t$ test was used to compare the means between two groups, and one-way analysis of variance (ANOVA) test was used to compare means of more than two groups. Mann Whitney test was used in stead of Student $t$ test in case of non parametric data.

- Pearson Chi square was used to compare percentages of qualitative data, and Fisher's Exact test was used for non parametric data.

- Pearson correlation test was used to compare two quantitative variables. The value of (r) is explained in the following figures:

$\mathrm{r}<0.2$ E negligible correlation

r 0.2-0.4 Ë weak correlation

r 0.4-0.7 $\ddot{E}$ moderate correlation

r 0.7-1 Ë strong correlation

r positive $\ddot{E}$ positive correlation

$r$ negative $\ddot{E}$ negative correlation

- For all these tests, the level of significance (P-value) can be explained as:

$\circ$ No significance $\mathrm{P}>0.05$

$\circ$ Significance $\mathrm{P}<0.05$

○ High significance $\mathrm{P}<0.001$.

\section{Results}

As regard of gender, our study results show that $60 \%$ of controls were females compared to $44.4 \%$ of cases with no significant difference. The mean age of the study groups is around 48 years in controls group and 53 years in cases group with nonsignificant difference.All of cases are due to HCV.All of cases have normal pulse rate.5 cases $(11.1 \%)$ had jaundice whileall of controls had nojaundice.8cases $(17.7 \%)$ had epistaxis.Non of the controls had epistaxis.Around half of the cases had no ascites, while $33.3 \%$ had mild ascites and $20 \%$ had moderate ascites. Non of the controls had ascites. The difference between cases and controls was significant.The majority of cases had no HE, with percentage of $64.4 \%$. It shows that 13 cases had grade 1 with percentage of $28.9 \%$. It shows that 3 cases are grade 2 with percentage of $6.7 \%$. 7cases $(15.5 \%)$ had dyspnea while all of controls had no dyspnea. 12 cases $(26.6 \%)$ had lower limbs oedema.

As regard investigations, the mean serum bilirubin of the study groups is around 0.91 in control group and 1.82 in case group. The mean albumin of the study groups is around 3.85 in control group and 3.07 in case group. The mean PT of the study groups is around 12.91 in control group and 16.30 in case group. The mean INR is around 1.11 in control group and 1.399 in case group.The mean serum haemoglobin of study group is around12.1 in control group and 9.9 in case group.The mean serum ALTofthe study groups is around 32.6 in control group and 40.8 in case group. The mean serum AST of the study groups is around32.2in control group and46.1 in case group.All of these investigations shows highly significant differences between cases and controls $(\mathrm{p}<0.001)$. On the other hand, the mean serum creatinine of the study groups is around 1.003 in control group and 1.02 in case group, with non-significant difference $(\mathrm{p}=0.691)$. 
Regarding echocardiographic data of the study groups, the mean left ventricleend diastolic diameter (LVEDD)of the study groups is around 4.91 $\pm 0.57 \mathrm{in}$ Control group and $4.83 \pm 0.63$ in Case group, with non-significant difference. It shows that the mean left ventricleend systolic diameter (LVESD) of the study groups is around 2.94 \pm 0.398 in Control group and $2.91 \pm 0.47$ in Case group, with nonsignificant difference. The mean right ventricle diameter (RVD) of the study groups is around $2.41 \pm 0.398$ in Control group and $2.55 \pm 0.42$ in Case group, with nonsignificant difference.It shows that the mean right atrium diameter (RAD) of the study groups is around $3.66 \pm 0.74$ in Control group and $3.46 \pm 0.83$ in Case group, with nonsignificant difference. The mean ejection fraction $(\mathrm{EF})$ of the study groups is around $69.67 \pm 3.11 \% \%$ in Control group and $67.92 \pm 5.44 \%$ in Case group, with nonsignificant difference (Table 1).

E/A ratio "represents the ratio of peak velocity flow in early diastole (the $\mathrm{E}$ wave) to peak velocity flow in late diastole caused by atrial contraction (the A wave)" $<1$ was significantly more in patients with LC than among controls ( $\mathrm{p}=0.035$ ).Our study results show that 15 cases Child Pugh Grade was A with percentage of $33.3 \%, 15$ cases Child Pugh Grade was B with percentage of 33.3\%,15 cases Child Pugh Grade wasCwith percentage of 33.3\%.MELD score ranged from8.5 to 10.4 , with a mean of 9.59 and a standard deviation of around 0.2 . The results show that only $22 \%$ of cases had no diastolic dysfunction, compared to $67 \%$ of controls. Also, $13.3 \%$ of cases had grade 2diastolic dysfunction, compared to zero among controls. The difference was statistically significant (Table2).

The LVEDD mean is $4.9 \pm 0.8$ in grade A Child-Pugh, $4.9 \pm 0.6$ in grade B and $4.6 \pm 0.5$ in grade $C$ with non-significant differences. It shows that the LVESD mean is $2.9 \pm 0.4$ in grade $A, 2.9 \pm 0.5$ in grade $B$ and $2.8 \pm 0.5$ in grade $C$ with non-significant value. Left atrium diameter (LAD)mean is $3.6 \pm 0.7$ in grade $A, 3.3 \pm 0.5$ in grade $B$ and $3.3 \pm 0.6$ in grade $\mathrm{C}$ with non-significant value. The RAD mean is $3.8 \pm 0.9$ in grade $\mathrm{A}$, $3.4 \pm 0.9$ in grade $B$ and $3.1 \pm 0.5$ in grade $C$ with non-significant value. The EF\% mean is $68 \pm 4.7$ in grade A, $68.9 \pm 5.1$ in grade $B$ and $66.9 \pm 6.5$ in grade $C$ with nonsignificant value. The mitral $\mathrm{E} / \mathrm{A}$ ratio is $1.09 \pm 0.5$ in grade $\mathrm{A}, 0.85 \pm 0.2$ in grade $\mathrm{B}$ and $0.88 \pm 0.3$ in grade $\mathrm{C}$, with non significant difference. On the other hand, Right ventricular diameter (RVD)mean is $2.4 \pm 0.4$ in grade A, $2.5 \pm 0.4$ in grade B and $2.8 \pm 0.8$ in grade $\mathrm{C}$ with significant difference (P value 0.018 ) (Table 3).Diastolic dysfunction was significantly higher among Child class $\mathrm{B}$ and $\mathrm{C}$ compared to A $(\mathrm{p}=0.020)$ ( Table 4).Although MELD increases steadily withsecond gradediastolic dysfunction, the difference was non significant (Table 5).Diastolic dysfunction was more common among patients withlarger amont of ascites, with significant difference.HE was significantly associated withdiastolic dysfunction.

Table 1 : Echocardiography data of studied group.

\begin{tabular}{lcccc}
\hline & Control & Case & T Test & P Value \\
\hline LVEDD(cm) & $4.91 \pm 0.57$ & $4.83 \pm 0.63$ & 0.437 & 0.664 \\
LVESD(cm) & $2.94 \pm 0.398$ & $2.91 \pm 0.47$ & 0.239 & 0.812 \\
LAD(cm) & $3.37 \pm 0.57$ & $3.35 \pm 0.61$ & 0.074 & 0.941 \\
RVD(cm) & $2.41 \pm 0.398$ & $2.55 \pm 0.42$ & 1.081 & 0.284 \\
RAD(cm) & $3.66 \pm 0.74$ & $3.46 \pm 0.83$ & 0.849 & 0.399 \\
EF\% & $69.67 \pm 3.11 \%$ & $67.92 \pm 5.44 \%$ & 1.183 & 0.242 \\
E/A & $1.174 \pm 0.394$ & $0.942 \pm 0.349$ & 2.158 & $\mathbf{0 . 0 3 5}$ \\
E/A $<\mathbf{1}$ & $5(33.3 \%)$ & $29(64.4 \%)$ & $4.434 *$ & $\mathbf{0 . 0 3 5}$ \\
\hline \multicolumn{5}{c}{ * Chi square test was used }
\end{tabular}


Table 2: Diastolic Dysfunction of studied group versus control group.

\begin{tabular}{|c|c|c|c|c|}
\hline & & & & Total \\
\hline \multirow{4}{*}{$\begin{array}{l}\text { Diastolic } \\
\text { Dysfunction }\end{array}$} & & Control & Case & \\
\hline & Normal & $10(66.7 \%)$ & $10(22.2 \%)$ & $20(33.3 \%)$ \\
\hline & Grade 1 (reversed G/A & $5(33.3 \%)$ & $29(64.4 \%)$ & $34(56.7 \%)$ \\
\hline & Grade 2 & 0 & $6(13.3 \%)$ & $6(10 \%)$ \\
\hline
\end{tabular}

Table 3: Echocardiographic data among different Child Pugh classes

\begin{tabular}{|c|c|c|c|c|c|}
\hline \multicolumn{6}{|c|}{ Child Pugh Class } \\
\hline & $A$ & $B$ & $C$ & Anova test & P value \\
\hline$L V E D D(\mathrm{~cm})$ & $4.9 \pm 0.8$ & $4.9 \pm 0.6$ & $4.6 \pm 0.5$ & 1.307 & 0.281 \\
\hline $\operatorname{LVESD}(\mathrm{cm})$ & $2.9 \pm 0.4$ & $2.9 \pm 0.5$ & $2.8 \pm 0.5$ & 0.463 & 0.633 \\
\hline$L A D(c m)$ & $3.6 \pm 0.7$ & $3.3 \pm 0.5$ & $3.3 \pm 0.6$ & 1.123 & 0.335 \\
\hline$R V D(\mathrm{~cm})$ & $2.4 \pm 0.4$ & $2.5 \pm 0.4$ & $2.8 \pm 0.4$ & 4.437 & $0.018(S)$ \\
\hline$R A D(\mathrm{~cm})$ & $3.8 \pm 0.9$ & $3.4 \pm 0.9$ & $3.1 \pm 0.5$ & 2.551 & 0.090 \\
\hline$E F(\%)$ & $68 \pm 4.7$ & $68.9 \pm 5.1$ & $66.9 \pm 6.5$ & 0.498 & 0.611 \\
\hline$E / A$ & $1.088 \pm 0.5$ & $0.854 \pm 0.2$ & $0.885 \pm 0.3$ & 2.082 & 0.137 \\
\hline
\end{tabular}

Table 4 : Grades of diastolic dysfunction and its relation to Child Pugh class Child class Total

\begin{tabular}{cccccc}
\hline & & A & B & C & \\
\hline Diastolic & Normal & $\mathbf{6 ( 4 0 \% )}$ & $\mathbf{3}(\mathbf{2 0 \%})$ & $\mathbf{1 ( 6 . 7 \% )}$ & $\mathbf{1 0}(\mathbf{2 2 . 2 \%})$ \\
Dysfunction & Grade 1 & $\mathbf{8}(\mathbf{5 3 . 3 \%})$ & $\mathbf{1 2}(\mathbf{8 0 \%})$ & $\mathbf{9 ( 6 0 \% )}$ & $\mathbf{2 9}(\mathbf{6 4 . 4 \%})$ \\
& Grade 2 & $\mathbf{1}(\mathbf{6 . 7 \%})$ & $\mathbf{0}$ & $\mathbf{5 ( 3 3 . 3 \% )}$ & $\mathbf{6 ( 1 3 . 3 \% )}$ \\
\hline \multicolumn{6}{c}{ Chi square $=11.697, \mathrm{p}$ value $=0.020(\mathrm{~S})$}
\end{tabular}

Table 5 : Grades of diastolic dysfunction and its relation to MELD score

\begin{tabular}{lc}
\hline Diastolic Dysfunction & MELD \\
\hline Normal & 9.72 \\
Grade 1 & 9.6 \\
Grade 2 & 9.9 \\
Total & 9.74 \\
\hline
\end{tabular}

\section{Discussion}

In the last 20 years, a number of evidences suggestedthat cirrhosis regardless of its etiology, is associated with the development of hemodynamic changes and major cardiovascular anomalies Overall these alterations are known as cirrhotic cardiomyopathy ${ }^{(\mathbf{1}}$, 2). Recognition of cirrhotic cardiomyopathy will depend on a high level of awareness and potentially will help better management of patients with cirrhosis ${ }^{(16)}$.Our study shows that the mean LVEDD of the study groups is around $4.91 \pm 0.57$ (in cm) in Control group and $4.83 \pm 0.63$ (in $\mathrm{cm}$ ) in Case group, with non-significant difference. While in Torregrosa et al., (2005) ${ }^{(17)}$ study the LVEDD was $4.8 \pm 0.5$ (in $\mathrm{cm}$ ) in patients without ascites, $4.6 \pm$ 0.5 (in $\mathrm{cm}$ ) in patients with ascites and $4.8 \pm 0.3$ (in $\mathrm{cm}$ ) in controls.In Karagiannakis et al., (2014) ${ }^{(18)}$ the 
mean LVEDD is with normal diastolic cardiac function was $5.15 \pm 0.46$ (in $\mathrm{cm})$ and patients with LVDD was $4.9 \pm$ 0.48 (in $\mathrm{cm}$ ) with no significant difference.

Our study shows that the mean LVESD of the study groups is around $2.94 \pm 0.398$ (in cm) in Control group and 2.91 \pm 0.47 (in $\mathrm{cm}$ ) in Case group, with non-significant difference. While in Torregrosa et al., (2005) ${ }^{(\mathbf{1 7})}$ study the LVE SD was $2.8 \pm 0.3$ (in $\mathrm{cm}$ ) in patients without ascites, $2.4 \pm 0.5$ (in $\mathrm{cm})$ patients with ascites and $2.9 \pm 0.3$ (in $\mathrm{cm}$ ) in controls. In Karagiannakis et al., (2014) ${ }^{(18)}$ the mean LVESD is with normal diastolic cardiac function was $5.15 \pm 0.46$ (in $\mathrm{cm}$ ) and patients with LVE DD was $4.9 \pm 0.48$ (in $\mathrm{cm}$ ) with no significant difference. In the Dahl et al., (2014) study the LVESD was $2.38 \pm 1.32$ (in $\mathrm{cm}$ ) in the group $\mathrm{A}$ patient (Child grade A), $3.29 \pm 0.91$ (in $\mathrm{cm}$ ) in the group B patients (Child grade B) and $2.72 \pm 1.24$ (in $\mathrm{cm}$ ) in the control group.Our study showsthat the mean LAD of the study groups is around $3.37 \pm 0.57$ (in $\mathrm{cm}$ ) in control group and $3.35 \pm 0.61$ (in $\mathrm{cm}$ ) in case group, with non-significant difference. While in Torregrosa et al., (2005) ${ }^{(17)}$ study the LAD was $3.9 \pm 0.6$ (in $\mathrm{cm}$ ) in patients without ascites, 4.3 \pm 0.6 (in $\mathrm{cm}$ ) patients with ascites and $3.9 \pm 0.4$ (in cm) in controls. This Study shows that the mean $\mathrm{EF}$ of the study groups is around $69.67 \pm 3.11 \%$ in Control group and $67.92 \pm 5.44 \%$ in Case group, with non- significant difference. While in the Torregrosa et al.,(2005) ${ }^{(17)}$ the EF was $73 \pm 6 \%$ in patientsand $65 \pm 4 \%$ in controls. In Karagiannakis et al.,(2014) ${ }^{(34)}$ the mean LVEF with normal diastolic cardiac function was $66.4 \pm 4.8 \%$ and patients with LVDD was $64.3 \pm$ $4.5 \%$ with no significant difference.

In the Dahl et al.,(2014) ${ }^{(19)}$ study the EF was $68.1 \pm$ percentage of $25 \%$ in alcoholic cirrhosis and 7 cases with percentage of $35 \%$ in nonalcoholic cirrhosis. There also were 9 cases with percent age of $45 \%$ in alcoholic cirrhosis and 7 cases with percentage of $35 \%$ in non-alcoholic cirrhosis. In Karagiannakis et al., (2014) $^{(18)}$ study about 26 cases Child Pugh grade was A with percentage of $59 \%, 15$ cases Child Pugh grade was B with percentage of $34 \%$ and 3 cases Child Pugh grade was $\mathrm{C}$ with percentage of $7 \%$. The Dahl et al.,(2014) ${ }^{(19)}$ study shows that the Child Pugh grade A was 12 with percentage of $63.2 \%$ and 7 were B with percentage of $36.8 \%$. Finally, the Serste et al., (2011) ${ }^{(20)}$ results show that the Child Pugh grade B was 2 with percentage of $20 \%$ and 8 were $\mathrm{B}$ with percentage of $80 \%$. In our study, only $22 \%$ of cases had no diastolic dysfunction, compared to $67 \%$ of controls. Also, $13.3 \%$ of cases had grade 2 diastolic dysfunction, compared to zero among controls. The difference was statistically significant. Also, diastolic dysfunction was significantly higher among Child class $\mathrm{B}$ and $\mathrm{C}$ compared to $\mathrm{A}(\mathrm{p}=0.02)$. On the other hand, although MELD increases steadily with diastolic dysfunction grade 2, the difference was non significant. Also, diastolic dysfunction was not statistically associated with age, serum bilirubin, PT, INR and serum creatinine. On the other hand, albumin level decreases steadily with diastolic dysfunction, with significant difference $(\mathrm{p}=0.011)$. Ascites in our study showed significant relation with diastolic dysfunction, while HE showed highly significant association with diastolic dysfunction ( $\mathrm{p}=0.004$, and $\mathrm{p}<0.001$; respectively). The Torregrosa et al.,(2005) ${ }^{(17)}$ study shows that in cirrhotic patients altered diastolic function at rest was observed in ascetic patients (elevated LAD and EDT). Karagiannakis et al.,(2014) ${ }^{(18)}$ study DD was found in 17 (38\%) of 
45 patients. Its presence was not found to be associated with the etiology and stage of cirrhosis, but its severity was directly correlated with the Child-Pugh score. The Dahl et al.,(2014) ${ }^{(19)}$ study shows that diastolic dysfunction is caused by decreased LV compliance and relaxation with an abnormal filling pattern of the ventricles. As, PFR (ewaves) was prolonged in cirrhotic patients compared to controls, whereas time to peak filling and peak ejection rate did not differ significantly between cirrhosis and controls.

\section{Conclusion}

Our study concluded that diastolic dysfunction is an important pathological complication associated with liver cirrhosis and is directly related to the severity of liver cirrhosis.

\section{References}

1.Ma Z, Lee SS. Cirrhotic cardiomyopathy: getting to the heart of the matter. Hepatology. 1996;24(2):451-9.

2.Zardi EM, Abbate A, Zardi DM, Dobrina A, Margiotta D, Van Tassell BW, et al. Cirrhotic cardiomyopathy. Journal of the American College of Cardiology. 2010;56(7):539-49.

3.Wong F. Cirrhotic cardiomyopathy. Hepatol Int. 2009;3:294-304.

4.Murphy SL, Xu J, Kochanek KD. Deaths: final data for 2010. Centers for Disease Control and Prevention website www cdcgov/nchs/data/nvsr/nvsr61/nvsr61_0 4 pdf 2014.

5.Blachier M, Leleu $\mathrm{H}$, PeckRadosavljevic M, Valla DC, RoudotThoraval F. The burden of liver disease in Europe: a review of available epidemiological data. Journal of hepatology. 2013;58(3):593-608.

6.Lok ASF, McMahon BJ. Chronic hepatitis B: update 2009. . Hepatology American Association for the Study of Liver Diseases website. 2014 (www.aasld.
org/practiceguidelines/Documents/Boo kmarked\% 20Practice \% 20Guidelines/Chronic_Hep_B_Update _2009\%20 8_24_2009.pdf.).

7.Castera L. Noninvasive methods to assess liver disease in patients with hepatitis B or C. Gastroenterology. 2012;142(6):1293-302 e4.

8.Fede G, D'Amico G, Arvaniti V, Tsochatzis E, Germani G, Georgiadis $\mathrm{D}$, et al. Renal failure and cirrhosis: a systematic review of mortality and prognosis. Journal of hepatology. 2012;56(4):810-8.

9.Brodehl A, Hedde PN, Dieding M, Fatima A, Walhorn V, Gayda S, et al. Dual color photoactivation localization microscopy of cardiomyopathyassociated desmin mutants. The Journal of biological chemistry. 2012;287(19):16047-57.

10.Moller S, Henriksen JH. Cardiovascular complications of cirrhosis. Gut. 2008;57(2):268-78

11.Moller S, Bendtsen F. Cirrhotic Multiorgan Syndrome. Digestive diseases and sciences. 2015;60(11):3209-25.

12.Chayanupatkul M, Liangpunsakul S. Cirrhotic cardiomyopathy: review of pathophysiology and treatment. Hepatol Int. 2014;8(3):308-15.

13.Lima JA, Desai MY. Cardiovascular magnetic resonance imaging: current and emerging applications. Journal of the American College of Cardiology. 2004;44(6):1164-71.

14.Pall A, Czifra A, Vitalis Z, Papp M, Paragh G, Szabo Z. Pathophysiological and clinical approach to cirrhotic cardiomyopathy. Journal of gastrointestinal and liver diseases : JGLD. 2014;23(3):301-10.

15.Pozzi M, Grassi G, Ratti L, Favini G, Dell'Oro R, Redaelli E, et al. Cardiac, neuroadrenergic, and portal hemodynamic effects of prolonged aldosterone blockade in postviral child A cirrhosis. The American journal of gastroenterology. 2005;100(5):1110-6. 
16. Mota VGa, B F, Markman. . Echocardiography in Chronic Liver Disease: Systematic Review. . Arq Bras Cardiol, . 2013;100(4)::376-85.

17.Torregrosa M, Aguade S, Dos L, e ta, $\mathrm{l}$. - Cardiac alterations in cirrhosis: reversibility after liver transplantation. Journal of hepatology. 2005;42:68-74.

18. Karagiannakis DS. Diastolic cardiac dysfunction is a predictor of dismal prognosis in patients with liver cirrhosis. . Hepatol Int 2014;8(4): :58894. .

19.Dahl EK. Diastolic and autonomic dysfunction in early cirrhosis: a dobutamine stress study. . Scand J Gastroenterol 2014;49(3): :362-72. .

20.Serste T. Beta-blockers cause paracentesis-induced circulatory dysfunction in patients with cirrhosis and refractory ascites: a cross-over study. . Journal of hepatology. 2011;55(4): :794-9. 
